\title{
Evaluation of synbiotics as gut health improvement agents against Shiga toxin-producing Escherichia coli isolated from the pig
}

Bo-Ra Kim ${ }^{1 \#}$, Kyung Jin $\mathrm{Cho}^{2 \#}$, Doowan $\mathrm{Kim}^{3 \#}$, Jin Ho Cho ${ }^{4 \#}$, Jun Hyung Lee ${ }^{1}$, Robin B. Guevarra ${ }^{1}$, Sun Hee Lee ${ }^{1}$, Jung Sun Kang ${ }^{2}$, Won Tak Cho ${ }^{2}$, Suphot Wattanaphansak ${ }^{5}$, Bit Na Kang ${ }^{6}$, Jong Nam Kim, Minho Song ${ }^{8^{*}}$ and Hyeun Bum Kim ${ }^{1^{*}}$

\author{
1 Department of Animal Resources Science, Dankook University, Cheonan 31116, Korea \\ ${ }^{2}$ Genebiotech Co., Ltd., Gongju 32619, Korea \\ ${ }^{3}$ National Institute of Animal Science, Rural Development Administration, Wanju 55365, Korea \\ ${ }^{4}$ Division of Food and Animal Sciences, Chungbuk National University, Cheongju 28644, Korea \\ ${ }^{5}$ Department of Veterinary Medicine, Faculty of Veterinary Science, Chulalongkorn University, Bangkok, Thailand \\ ${ }^{6}$ Abbvie Bioresearch Center, Abbvie, Worcester, MA, USA \\ ${ }^{7}$ Department of Beef Science, Korea National College of Agriculture and Fisheries, Jeonju 54874, Korea \\ ${ }^{8}$ Division of Animal and Dairy Science, Chungnam National University, Daejeon 34134, Korea
}

\begin{abstract}
Colibacillosis is one of the major health problems in young piglets resulting in poor health and death caused by Escherichia coli producing F18 pili and Shiga toxin 2e. It is pivotal to reduce colibacillosis in weaned piglets to enhance production performance. In this study, we evaluated synbiotics as the gut health improvement agents in the mouse model challenged with Shiga toxin-producing E. coli (STEC) isolated from piglets. Prebiotic lactulose was formulated with each $5.0 \times 10^{6} \mathrm{CFU} / \mathrm{mL}$ of Pediococcus acidilactici GB-U15, Lactobacillus plantarum GB-U17, and Lactobacillus plantarum GB 1-3 to produce 3 combinations of synbiotics. A total of 40 three weeks old BALB/c mice were randomly assigned to 4 groups $(n=10)$ : a control group and 3 synbiotics treated groups. Each treatment groups were daily administrated with $5.0 \times 10^{6} \mathrm{CFU} / \mathrm{mL}$ of one synbiotics for the first week, and every 3 days during the second week. All the mice were challenged with $8.0 \times 10^{8} \mathrm{CFU} / \mathrm{mL}$ of STEC 5 days after animals began to receive synbiotics. Mice treated with synbiotics based on Pediococcus acidilactici GBU15 and Lactobacillus plantarum GB-U17 significantly improved daily weight gain compared to mice in other groups. While mice treated with GB-U15 showed better fecal index, no significant differences were observed among groups. Gross lesion and histopathological evaluations showed that mice treated with GB-U15 moderately improved recovery from STEC infection. In conclusion, our results suggest that the synbiotics formulated with lactulose and Pediococcus acidilactici GB-U15 have potential benefits to prevent and improve colibacillosis in weaned piglets.
\end{abstract}

Keywords: Escherichia coli, Gut health, Pig, Synbiotics

\footnotetext{
Received: Jan 21, 2019 Revised: Jan 28,2019 Accepted: Jan 29, 2019

"These authors contributed equally to this work.

"Corresponding author: Minho Song, Division of Animal and Dairy Science, Chungnam National University, Daejeon 34134, Korea.

Tel: +82-42-821-5446, E-mail: mhsong@cnu.ac.kr

Hyeun Bum Kim, Department of Animal Resources Science, Dankook University, Cheonan 31116, Korea.

Tel: +82-41-550-3653, E-mail: hbkim@dankook.ac.kr
}

This is an Open Access article distributed under the terms of the Creative Commons Attribution Non-Commercial License (http://creativecommons.org/licenses/by$\mathrm{nc} / 4.0 /$ ) which permits unrestricted non-commercial use, distribution, and reproduction in any medium, provided the original work is properly cited.

Copyright (C) 2019 Korean Society of Animal Science and Technology. 


\section{Background}

Diarrhea is one of the major causes of mortality in neonatal and young piglets. It attributes to the approximately half (49\%) of the pig death [1]. One of the most detrimental diseases resulting in diarrhea and death in swine is colibacillosis that is caused by pathogenic Escherichia coli (E. coli) infection [2]. The main symptoms of colibacillosis include vomiting, severe diarrhea, and dehydration leading to high mortality [2]. Though it occurs in pigs regardless of age, young piglets under 1 week of age are most vulnerable [3]. E. coli infection is transmitted mostly by the oral route through feed or water contaminated with pathogenic E. coli. In the case of newborn piglets, the infection is commonly transmitted through the feces or the nipple [3].

As the preventive and therapeutic measures against colibacillosis, antibiotics, such as antibiotic growth promoters (AGPs), have long been used. Therefore, the stable supply of effective antibiotics for pigs has been thought to be essential for the enhanced health and well-being of the animals [4]. As such, approximately 1,000 tons of macrolide and tetracycline antibiotics alone were added to pig feed each year in the U.S [5]. Despite the beneficial effects of antibiotics in pig production, there is a concern that the use of antibiotics in food animals may lead to the selection for antibiotic-resistant bacteria and the spread of increased antibiotic resistance gene pool in commensal bacteria in humans as well as animals [4].

In addition, the long-term use of antibiotics in agriculture makes it difficult to cure animal diseases caused by bacteria that are highly fertile and resistant [6]. Because the risks of antibiotics usage outweigh cost savings in livestock production [7], many countries have limited the use of antibiotics, and banned AGPs in food animal production. Consequently, probiotics, prebiotics or synbiotics have been presented as the most desirable alternatives to antibiotics due to their beneficial effects [8].

Probiotics are feed additives that can be used to replace antibiotics in animal feed [9]. They refer to a group of beneficial bacteria that contribute to the health and well-being of the host when administered in sufficient amounts [3]. As an antibiotic substitute, probiotics enhance the productivity of livestock by improving resistance to pathogens, helping the growth of beneficial microbiota in the gut and boosting immune activity. Prebiotics, such as lactulose, oligosaccharides, and lactitol are non-digestible feed supplements with constructive effects on the host as they assist the growth and activity of beneficial microbiota in the gut [9]. Prebiotics can selectively stimulate the growth of beneficial microbiota in the gut, therefore improve the immunity of livestock and durably maintain intestinal microbiota. Synbiotics, a mixture of probiotics and prebiotics, were developed to further enhance the effects of probiotics. Synbiotics, are ingested as feed supplements to provide health ben- efits through synergistic effects [10]. One mechanism of actions of synbiotics is to enhance the intestinal attachment of probiotics resulting in improved livestock productivity and feed efficiency $[11,12]$. As demand for synbiotics continuously grows because of their beneficial effects on livestock health, there are immense needs to develop synbiotics. Therefore, in this study, we produced the stable quality feed additives, synbiotics, and evaluated synbiotics as gut health improvement agents in the mouse model challenged with Shiga toxin-producing E. coli (STEC) isolated from piglets.

\section{Materials and Methods}

\section{Animals and experimental design}

A total of 40 3-week old BALB/c mice were purchased from RaonBio Inc. (Gyeonggi-do, Korea), and randomly assigned into four groups $(n=10)$ : control group without any treatment, treatment group 1 treated with synbiotics based on Pediococcus acidilactici GB-U15, treatment group 2 treated with synbiotics based on Lactobacillus plantarum GB-U17, and treatment group 3 treated with synbiotics based on Lactobacillus plantarum GB 1-3. Each synbiotics treated groups were daily administrated with $5.0 \times 10^{6} \mathrm{CFU} /$ $\mathrm{mL}$ of one synbiotics for the first week, and every 3 days during the second week. Mice were challenged with $8.0 \times 10^{8} \mathrm{CFU} / \mathrm{mL}$ of STEC 5 days after animals began to receive synbiotics. Mice were reared in confinement with controlled light, temperature $\left(25^{\circ} \mathrm{C} \pm 2\right)$. All the mice in this experiment were fed with the same feed ad libitum. The general health status of mice was evaluated by recording fecal index, daily weight gain, mortality and clinical symptoms. Fecal index criterions that indicate stool hardness or softness are as follows. 1: Hard, dry pellets in a small, hard mass, 2: Hard, formed stool that remains firm and soft, 3: Soft, formed and moist stool that retains its shape, 4: Soft, unformed stool that assumes the shape of the container, 5: Watery, liquid stool that can be poured.

\section{Source of synbiotics}

Prebiotics, lactulose, was formulated with each $5.0 \times 10^{6} \mathrm{CFU} /$ $\mathrm{mL}$ of Pediococcus acidilactici GB-U15, Lactobacillus plantarum GBU17, and Lactobacillus plantarum GB 1-3, to produce 3 types of synbiotics. Probiotics were randomly selected lactic acid producing strains through evaluation of antimicrobial activity in vitro, and selected strains had excellent inhibitory effects against STEC, which is one of the major health problems in young piglets resulting in poor health and death. Three synbiotics containing lactulose and one of each Pediococcus acidilactici GB-U15, Lactobacillus plantarum GB-U17, and Lactobacillus plantarum U1-3 were manufactured by the Genebiotech Co., Ltd. (Seoul, Korea). Each synbiotics contains at least $5.0 \times 10^{6} \mathrm{CFU} / \mathrm{mL}$ of one probiotics selected. 


\section{Oral challenge}

STEC JOL576 was incubated in Luria-Bertani (LB) Broth (LPS SOLUTION, Daejeon, Korea) for 48 hours at $37^{\circ} \mathrm{C}$. All the mice ( $\mathrm{n}=5$ per group) were challenged with $150 \mathrm{uL}$ of bacterial culture media containing $8.0 \times 10^{8} \mathrm{CFU} / \mathrm{mL}$ of STEC by oral gavage.

\section{Tissue sampling and histological analysis}

Two mice in each group were sacrificed by cervical dislocation one week after STEC oral challenge, and all other individual mice were autopsied at the end of the experiment, two weeks after STEC oral challenge. For histological examination, ileum and colon samples were fixed in 10\% neutral buffered formalin, and stained with hematoxylin and eosin (HE). The staining was carried out by the Abion Inc. (Seoul, Korea).

\section{Statistical analysis}

Statistical analysis was performed with General Linear Model Procedure of the SAS software v.9.3 (SAS Institute, USA) using the Tukey's honest significance test (HSD). Differences were considered significant at $p<0.05$.

\section{Results}

\section{Growth performance}

Mice treated with synbiotics based on Pediococcus acidilactici GB-
U15 and Lactobacillus plantarum GB-U17 significantly improved daily weight gain compared to mice in other groups. In particular, mice treated with synbiotics based on GB-U15 showed the best growth performance (Table 1).

\section{Fecal index}

While mice treated with GB-U15 showed better fecal index, no significant differences were observed among groups (Fig. 1). Feces from the mice challenged with STEC were softer and more moisture compared to those of normal mice. It took a shorter time for the mice treated with synbiotics (group 1, 2, and 3) after ETEC challenge to return to the fecal index 1 than the mice without the synbiotics treatment.

\section{Gross lesion and histological analysis}

In all groups, the gross lesion and histopathological evaluations showed that the mice treated with synbiotics (group 1,2, and 3) moderately improved recovery from STEC infection compared to those without the synbiotics treatment. The mice treated with GB-U 15 showed generally improved recovery compared to the other groups treated with GB-U17 and GB 1-3. Also, gross lesion and histopathological evaluations showed that mice treated with GB-U15 moderately improved recovery from STEC infection compared to other groups of mice. However, there were no significant differences were observed (Fig. 2).

Table 1. Growth performance comparisons among treatment groups based on their average relative percent weight

\begin{tabular}{|c|c|c|c|c|c|c|c|c|c|}
\hline \multirow[t]{2}{*}{ DPI ${ }^{11}$} & \multicolumn{2}{|c|}{ Control $^{2)}$} & \multicolumn{2}{|c|}{ Group $1^{3)}$} & \multicolumn{2}{|c|}{ Group $2^{4)}$} & \multicolumn{2}{|c|}{ Group $3^{5)}$} & \multirow[t]{2}{*}{$p$-value } \\
\hline & Average & $\begin{array}{l}\text { Standard } \\
\text { error }\end{array}$ & Average & $\begin{array}{l}\text { Standard } \\
\text { error }\end{array}$ & Average & $\begin{array}{l}\text { Standard } \\
\text { error }\end{array}$ & Average & $\begin{array}{l}\text { Standard } \\
\text { error }\end{array}$ & \\
\hline 0 & $100^{a}$ & 0.00 & $100^{a}$ & 0.00 & $100^{a}$ & 0.00 & $100^{a}$ & 0.00 & \\
\hline 1 & $100.75^{\mathrm{b}}$ & 0.42 & $102.62^{\mathrm{ab}}$ & 0.68 & $103.45^{\mathrm{a}}$ & 0.50 & $101.50^{\mathrm{b}}$ & 0.18 & 0.004 \\
\hline 2 & $101.55^{\mathrm{b}}$ & 0.42 & $105.07^{\mathrm{a}}$ & 1.14 & $106.85^{\mathrm{a}}$ & 1.01 & $105.23^{\mathrm{a}}$ & 0.43 & 0.0016 \\
\hline 3 & $91.32^{\mathrm{b}}$ & 0.41 & $94.03^{\mathrm{ab}}$ & 0.72 & $94.42^{\mathrm{a}}$ & 1.29 & $92.72^{\mathrm{ab}}$ & 0.15 & 0.0402 \\
\hline 4 & $90.50^{\mathrm{a}}$ & 0.70 & $91.93^{\mathrm{a}}$ & 0.84 & $91.85^{\mathrm{a}}$ & 1.52 & $90.15^{\mathrm{a}}$ & 0.78 & 0.4999 \\
\hline 5 & $96.88^{\mathrm{a}}$ & 1.00 & $98.53^{\mathrm{a}}$ & 0.91 & $98.27^{\mathrm{a}}$ & 1.53 & $94.90^{\mathrm{a}}$ & 0.57 & 0.0924 \\
\hline 6 & $100.15^{b}$ & 0.17 & $103.05^{a}$ & 0.77 & $102.83^{a}$ & 0.72 & $99.75^{b}$ & 0.28 & 0.0014 \\
\hline 7 & $102.98^{\mathrm{b}}$ & 0.25 & $105.70^{a}$ & 0.72 & $105.65^{a}$ & 0.83 & $102.28^{b}$ & 0.39 & 0.0022 \\
\hline 8 & $103.18^{b}$ & 0.63 & $107.93^{\mathrm{a}}$ & 1.13 & $107.53^{\mathrm{a}}$ & 1.41 & $102.70^{b}$ & 0.50 & 0.0036 \\
\hline 9 & $105.40^{\mathrm{a}}$ & 0.68 & $109.83^{a}$ & 1.77 & $109.80^{a}$ & 1.56 & $106.50^{a}$ & 1.74 & 0.1303 \\
\hline 10 & $108.15^{\mathrm{ab}}$ & 0.62 & $112.10^{\mathrm{a}}$ & 1.62 & $111.10^{\mathrm{ab}}$ & 1.45 & $106.68^{b}$ & 0.50 & 0.0207 \\
\hline 11 & $107.25^{\mathrm{ab}}$ & 0.66 & $122.53^{\mathrm{a}}$ & 1.51 & $111.00^{\mathrm{ab}}$ & 1.89 & $106.43^{b}$ & 0.58 & 0.0159 \\
\hline 12 & $109.18^{b}$ & 0.55 & $114.85^{\mathrm{a}}$ & 1.81 & $114.60^{\mathrm{ab}}$ & 1.62 & $109.48^{\mathrm{ab}}$ & 0.78 & 0.0114 \\
\hline
\end{tabular}

${ }^{a, b}$ Average values in the same row with different superscripts are statistically different.

${ }^{1)} \mathrm{DPI}$, days post inoculation.

${ }^{2)}$ Control, group without any treatment.

${ }^{3)}$ Group 1, treated with synbiotic based on Pediococcus acidilactici GB-U15.

${ }^{4)}$ Group 2, treated with synbiotic based on Lactobacillus plantarum GB-U17.

${ }^{5)}$ Group 3, treated with synbiotic based on Lactobacillus plantarum GB1-3. 


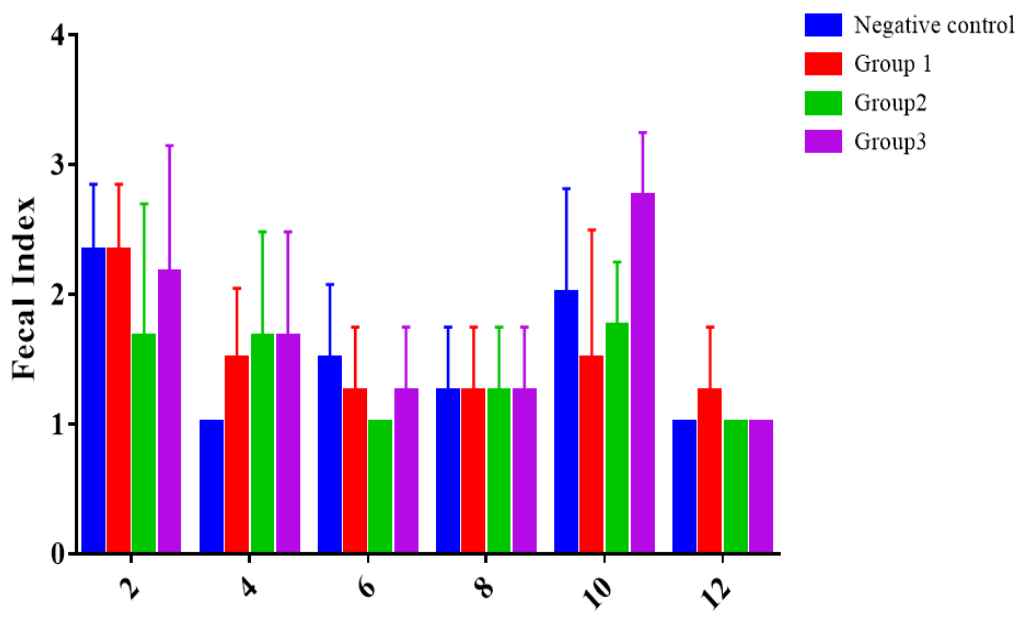

Day post-infection (DPI)

Fig. 1. Effects of oral STEC challenge and synbiotics' administration on the fecal consistency score of piglets. NC, control group without any treatment; Group 1, treated with synbiotic based on Pediococcus acidilactici GB-U15; Group 2, treated with synbiotic based on Lactobacillus plantarum GBU17; Group 3, treated with synbiotic based on Lactobacillus plantarum GB1-3.

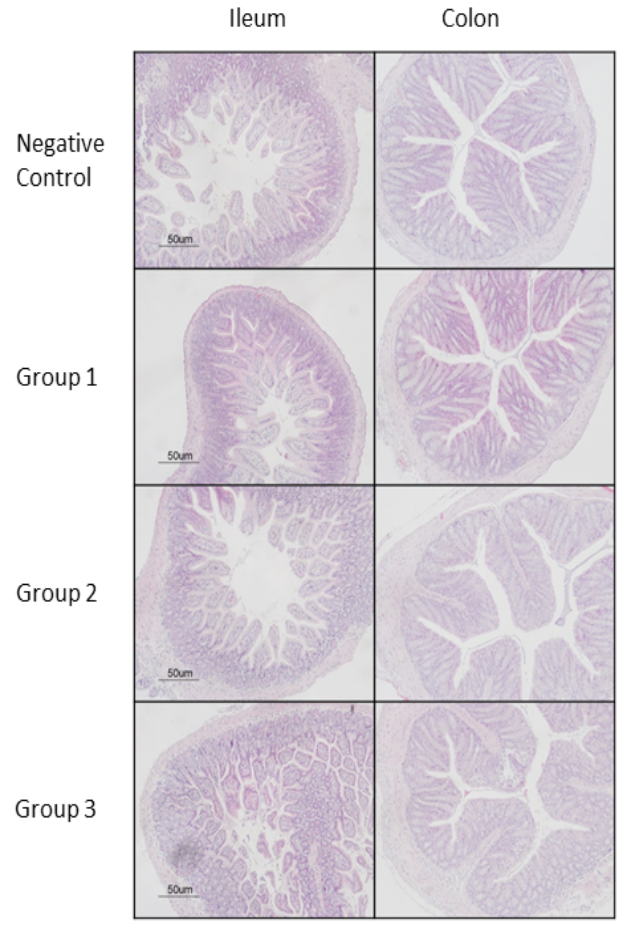

Fig. 2. Effects of the oral $E$. coli challenge and synbiotics' administration on the intestinal morphology of mice (100x magnification). Mild inflammation and minimal luminal debris were observed in the colons of all mice. NC, control group without any treatment; Group 1, treated with synbiotic based on Pediococcus acidilactici GB-U15; Group 2, treated with synbiotics based on Lactobacillus plantarum GB-U17; Group 3, treated with synbiotic based on Lactobacillus plantarum GB1-3.

\section{Discussion}

While the nutritional content of the animal feed is adjusted to optimize the impact on animal health and growth, feed additives including probiotics, prebiotics, and synbiotics are used to enhance livestock productivity $[7,13]$. When the host experiences high stress usually with a slower growth rate or weakened immune systems, probiotics are used to restore or strengthen natural gut microbial balance [14]. By improving intestinal microbial balance, probiotics including Lactobacilli and Bacilli heighten livestock productivity $[8,15,16]$. When prebiotics is used as feed supplements in livestock production, they assist the growth and activity of beneficial microbiota in the gut resulting in boosting the host's immune system [2]. A mixture form of probiotics and prebiotics, synbiotics, are commonly used to further enhance the effects of probiotics to provide livestock with health benefits through synergistic effects. Synbiotics selectively stimulate the passage of probiotic bacteria through the gut and help growth and/or colonization of the beneficial bacteria in the intestines [17]. As AGPs are limited or banned in livestock production worldwide, alternatives including probiotics, prebiotics and synbiotics are becoming more on demand in livestock farming because of their beneficial effects on animal health and well-being $[7,18,19]$.

Disparate studies have shown the beneficial effects of probiotics on pig growth performance and health $[20,21]$. The mixture of fructooligosaccharides and $L$. paracase $i$ has been shown to stimulate the growth of beneficial bacteria in the gut, to reduce the number of harmful bacteria including $E$. coli, and to improve the morphology of intestinal villi [22-24]. Other studies show that synbiotics are more effective when fed to suckling piglets [8,24]. While mortality decreased, the gut microbial diversity was increased [22]. It has been proved to be useful in maintaining host intestinal microflora and improving animal health [8]. As such, our results showed that mice treated with synbiotics based on Pediococcus acidilactici 
GB-U15 and Lactobacillus plantarum GB-U17 with lactulose significantly improved daily weight gain compared to mice in other groups. In particular, mice treated with synbiotics based on GBU15 showed the best growth performance. While mice treated with GB-U15 showed better fecal index, no significant differences were observed among groups. Gross lesion and histopathological evaluations showed that mice treated with GB-U15 moderately improved recovery from STEC infection. Similar to the results from various studies, our results suggest that the synbiotics formulated with lactulose and Pediococcus acidilactici GB-U15 has potential benefits to prevent and improve colibacillosis in weaned piglets.

However, the exact effects of synbiotics formulated with lactulose and Pediococcus acidilactici GB-U15 are still needed to be elucidated as the effects of probiotics and prebiotics on livestock are often incongruent among different studies [25]. Understanding of the precise mechanisms for proving the beneficial effects of synbiotics in animal health and well-being will also lead us to understand the unknown interactions between feed additives and intestinal flora $[7,16,20]$.

\section{Conclusion}

Our results suggest that the synbiotics formulated with lactulose and Pediococcus acidilactici GB-U15 have potential benefits to prevent and improve colibacillosis in weaned piglets.

\section{Competing interests}

No potential conflict of interest relevant to this article was reported.

\section{Funding sources}

The present study was supported by the Rural Development Administration (Project No. PJ012615) Republic of Korea and the Ministry of SMEs and Startups (MSS) under the "Regional Specialized Industry Development Program (R0004225)" supervised by the Korea Institute for Advancement of Technology (KIAT).

\section{Acknowledgements}

The authors thank Mo Re Kim (Westborough High School, MA, USA) for the English editing.

\section{Availability of data and material}

Upon reasonable request, the datasets of this study can be available from the corresponding author.

\section{Authors' contributions}

Conceptualization: Cho JH, Song M, Kim HB.

Formal analysis: Kim BR, Cho KJ, Kim DW, Cho JH, Song M, Kim HB.
Methodology: Kim BR, Cho KJ, Kim DW, Cho JH, Lee JH, Guevarra B. R, Lee SH, Kang JS, Cho WT, Wattanaphansak S, Kang BN, Kim JN, Song M, Kim HB.

Writing - original draft: Kim BR, Cho KJ, Kim DW, Cho JH, Lee JH, Guevarra B. R, Lee SH, Kang JS, Cho WT, Wattanaphansak S, Kang BN, Kim JN, Song M, Kim HB.

Writing - review \& editing: Kim BR, Cho KJ, Kim DW, Cho JH, Song M, Kim HB.

\section{Ethics approval and consent to participate}

This study was approved by IACUC of Dankook University (No. DKU-2015-113).

\section{ORCID}

Bo-Ra Kim

Kyung Jin Cho https://orcid.org/0000-0001-8824-5034

Doowan Kim

Jin $\mathrm{Ho}$ Cho

Jun Hyung Lee https://orcid.org/0000-0002-6698-3674 https://orcid.org/0000-0003-2392-5535 https://orcid.org/0000-0001-7151-0778 https://orcid.org/0000-0003-0637-8250

Robin B. Guevarra https://orcid.org/0000-0002-4809-1816 Sun Hee Lee https://orcid.org/0000-0002-3481-5633 Jung Sun Kang http://orcid.org/0000-0002-7489-5607 Won Tak Cho http://orcid.org/0000-0002-8063-805X Suphot Wattanaphansak https://orcid.org/0000-0003-2251-1314 Bit Na Kang Jong Nam Kim Minho Song https://orcid.org/0000-0001-5037-9205 https://orcid.org/0000-0002-8034-7156 Hyeun Bum Kim https://orcid.org/0000-0002-4515-5212 https://orcid.org/0000-0003-1366-6090

\section{References}

1. Yang $Q$, Huang $X$, Zhao S, Sun W, Yan Z, Wang P, et al. Structure and function of the fecal microbiota in diarrheic neonatal piglets. Front Microbiol. 2017;8:502.

2. Tran THT, Everaert N, Bindelle J. Review on the effects of potential prebiotics on controlling intestinal enteropathogens Salmonella and Escherichia coli in pig production. J Anim Physiol Anim Nutr. 2018;102:17-32.

3. Reid G, Jass J, Sebulsky MT, McCormick JK. Potential uses of probiotics in clinical practice. Clin Microbiol Rev. 2003;16:658-72.

4. Stanton TB. A call for antibiotic alternatives research. Trends Microbiol. 2013;21:111-3.

5. Apley MD, Bush EJ, Morrison RB, Singer RS, Snelson H. Use estimates of in-feed antimicrobials in swine production in the United States. Foodborne Pathog Dis. 2012;9:272-9.

6. Michael GB, Kadlec K, Sweeney MT, Brzuszkiewicz E, Liesegang H, Daniel R, et al. ICEPmu1, an integrative conjugative 
element (ICE) of Pasteurella multocida: analysis of the regions that comprise 12 antimicrobial resistance genes. J Antimicrob Chemother. 2012;67:84-90.

7. Allen HK, Levine UY, Looft T, Bandrick M, Casey TA. Treatment, promotion, commotion: antibiotic alternatives in food-producing animals. Trends Microbiol. 2013;21:114-9.

8. Meng QW, Yan L, Ao X, Zhou TX, Wang JP, Lee JH, et al. Influence of probiotics in different energy and nutrient density diets on growth performance, nutrient digestibility, meat quality, and blood characteristics in growing-finishing pigs. J Anim Sci. 2010;88:3320-6.

9. Gibson GR, Roberfroid MB. Dietary modulation of the human colonic microbiota: introducing the concept of prebiotics. J Nutr. 1995;125:1401-12.

10. Aureli P, Capurso L, Castellazzi AM, Clerici M, Giovannini M, Morelli L, et al. Probiotics and health: an evidence-based review. Pharmacol Res. 2011;63:366-76.

11. Soccol CR, Vandenberghe LPD, Spier MR, Medeiros ABP, Yamaguishi CT, Lindner JD, et al. The potential of probiotics: a review. Food Technol Biotech. 2010;48:413-34.

12. Piva A, Casadei G, Gatta PP, Luchansky JB, Biagi G. Effect of lactitol, lactic acid bacteria, or their combinations (synbiotic) on intestinal proteolysis in vitro, and on feed efficiency in weaned pigs. Can J Anim Sci. 2005;85:345-53.

13. Guevarra RB, Hong SH, Cho JH, Kim BR, Shin J, Lee JH, et al. The dynamics of the piglet gut microbiome during the weaning transition in association with health and nutrition. J Anim Sci Biotechnol. 2018;9:54.

14. Ahmed ST, Hoon J, Mun HS, Yang CJ. Evaluation of Lactobacillus and Bacillus-based probiotics as alternatives to antibiotics in enteric microbial challenged weaned piglets. Afr J Microbiol Res. 2014;8:96-104.

15. Djouzi Z, Andrieux C, Degivry MC, Bouley C, Szylit O. The association of yogurt starters with Lactobacillus casei DN 114.001 in fermented milk alters the composition and metabolism of intestinal microflora in germ-free rats and in human flora-associated rats. J Nutr. 1997;127:2260-6.

16. Alexopoulos C, Georgoulakis IE, Tzivara A, Kritas SK, Siochu
A, Kyriakis SC. Field evaluation of the efficacy of a probiotic containing Bacillus licheniformis and Bacillus subtilis spores, on the health status and performance of sows and their litters. J Anim Physiol Anim Nutr. 2004;88:381-92.

17. Roberfroid MB. Prebiotics and synbiotics: concepts and nutritional properties. Br J Nutr. 1998;80:S197-202.

18. Callaway TR, Edrington TS, Anderson RC, Harvey RB, Genovese KJ, Kennedy CN, et al. Probiotics, prebiotics and competitive exclusion for prophylaxis against bacterial disease. Anim Health Res Rev. 2008;9:217-25.

19. Guevarra RB, Lee JH, Lee SH, Seok MJ, Kim DW, Kang $\mathrm{BN}$, et al. Piglet gut microbial shifts early in life: causes and effects.J Anim Sci Biotechnol. 2019;10:1.

20. Bratz K, Golz G, Janczyk P, Nockler K, Alter T. Analysis of in vitro and in vivo effects of probiotics against Campylobacter spp. Berl Munch Tierarztl Wochenschr. 2015;128:155-62.

21. Zeyner A, Boldt E. Effects of a probiotic enterococcus faecium strain supplemented from birth to weaning on diarrhoea patterns and performance of piglets. J Anim Physiol Anim Nutr (Berl). 2006;90:25-31.

22. Nemcova R, Bomba A, Gancarcikova S, Herich R, Guba P. Study of the effect of Lactobacillus paracasei and fructooligosaccharides on the faecal microflora in weanling piglets. Berl Munch Tierarztl Wochenschr. 1999;112:225-8.

23. Bomba A, Nemcova R, Gancarcikova S, Herich R, Guba P, Mudronova D. Improvement of the probiotic effect of micro-organisms by their combination with maltodextrins, fructo-oligosaccharides and polyunsaturated fatty acids. Br J Nutr. 2002;88 Suppl 1:S95-9.

24. Shim SB, Verstegen MWA, Kim IH, Kwon OS, Verdonk JMAJ. Effects of feeding antibiotic-free creep feed supplemented with oligofructose, probiotics or synbiotics to suckling piglets increases the preweaning weight gain and composition of intestinal microbiota. Arch Anim Nutr. 2005;59:419-27.

25. Gaggia F, Mattarelli P, Biavati B. Probiotics and prebiotics in animal feeding for safe food production. Int J Food Microbiol. 2010;141 Suppl 1:S15-28. 